\title{
Research of 3D Body Models Computer Adjustment Based on Anthropometric Data Determined by Laser 3D Scanner
}

\author{
Slavenka PETRAK, Maja MAHNIC, Darko UJEVIC \\ Faculty of Textile Technology, University of Zagreb, Zagreb, Croatia
}

\begin{abstract}
The paper presents the investigation of the computer 3D body model adjustment possibilities for the implementation in the 3D CAD system for computer-aided design and garment simulation. In this context, three methods of connecting data on measurements and body shape found by using 3D body scanner and 2D/3D CAD system for computer-aided design and garment simulation were investigated. The first method refers to the research of the possibilities of interactive avatar adjustment in the CAD system based on the analysis of the anthropometric characteristics of the body and measurements found by using a 3D body scanner. The second method involves establishing a systematic record of body measurements found by using a 3D body scanner as the input record format in a 3D CAD system, which is used for automatic avatar adjustment. The third method involves creating a computer record of the body model using the program for 3D scanning, that is suitable for the direct implementation in the 3D CAD system for garment simulation.
\end{abstract}

Keywords: 3D body scanning, CAD system, parametric body model, clothing simulation

\section{Introduction}

The application of the 3D body scanner has an increasing implementation in the field of body measurement for garment construction in the last decade [1-3]. In addition to determine the linear body measurements that are most commonly used data in the clothing industry and on which conventional clothing construction is based, 3D scanning is used to obtain data on body shape, anthropometric relationships of individual body parts, deviations from the normal proportions, and body posture [4]. In this manner all relevant data necessary for computer-aided design and modification of garment patterns according to the individual body anthropometric characteristics are determined.

International standard ISO 20685 has been developed to ensure the comparability of body measurements determined by ISO 7250 (Basic Human Body Measurements for Technological Design) and ISO 8559 (Garment Construction and Anthropometric Surveys - Body Dimensions) using various $3 \mathrm{D}$ body scanners [5]. In this way all the anthropometric data determined using any 3D scanner can be suitable for the inclusion in an international database, such as those described in ISO 15535 (203, General requirements for establishing anthropometric databases) [6]. However, for performing 3D garment simulations by using the CAD system it is also necessary to ensure harmonization between the anthropometric measurements determined by 3D body scanner and the corresponding measurements that can be adjusted on the avatar in the CAD system.

Most existing computer programs for 3D virtual garment use parametric models of the human body with different number of body measurements or avatars, which can be interactively modified and adjusted [7]. In this context, the paper presents three methods for computer adaptations of the 3D body models in CAD system, based on anthropometric data determined by 3D scanning. Special attention is devoted to problems of the still existing discrepancy of anthropometric landmarks on the 3D body model determined using 3D scanner and avatar implemented into the CAD system. For the study described in the paper the Human Solutions 3D body scanner and OptiTex CAD system for garment design and simulation were used.

\section{Methods}

The OptiTex CAD system allows working with parametric body model using 49 various body measurements that can be adjusted and combined with each other.

In addition to interactive adjustments by entering each measurement this research investigates the possibility of automatic avatar adjustment by creating data files of systematically organized measurement sets determined by $3 \mathrm{D}$ scanning and their implementation in the appropriate record format in the 3D CAD system, and the possibility of direct implementation of computer model obtained by $3 \mathrm{D}$ body scanning. 


\subsection{Methodology of the Vitus Smart 3D laser body scanner operation}

The operation principle of laser scanners is based on the measurement of polar coordinates and the horizontal and vertical angle and distances to each space point, fig. 1. The instrument sends, according to the predefined interval, the sequence of laser pulses. Registering the total shift of the system in relation to its initial position and the length measured, the spatial coordinates of each point are determined. A 3D group of points is arranged in parallel, horizontal planes, with a point which amounting to about $1 \mathrm{~mm}$ in the horizontal plane and about $2 \mathrm{~mm}$ in the vertical plane. Each camera captures body segments and processes the data of the captured segment into the computer. Individual segments are computer-linked to a 3D group of points describing the shape of the body. Laser scanning opens the possibility of collecting large amounts of 3D data on the object to be captured. The set of points in the 3D coordinate system is called point cloud. For some applications, such as determining body measurements, it is sufficient to use data in their original form, without or with minimal additional processing. The measured point cloud allows instant measurements almost immediately using the computer without physical access to the real object or body.

For easier visualization and presentation it is enough to generate the topology of the measured objects (irregular areas) from the collected geometric data using automatic algorithms; in this manner very faithful models are obtained. In fact, all manufacturers of laser scanners with their equipment supply a computer program that allows the visualization and determination of body measurements, and the triangulation of 3D points where certain points are connected creating a surface network to visualize the object and can be further processed in some of the 3D programs. The triangulation process is carried out automatically, whereby the computer uses complex mathematical algorithms with respect to the distinct density of points that need to be connected, and the most complex part of the triangulation process is proper selection of points. Each of the points $(x, y, z)$ has its coordinates that define the position of points in the three-dimensional Cartesian coordinate system. The $x, y$ and $z$ coordinates are usually oriented in such a way that the $x$ and $y$ coordinates specify the plane where the object is, and the $z$ coordinate is oriented perpendicular to the plane $(x, y)$ in the direction of the height of the object.

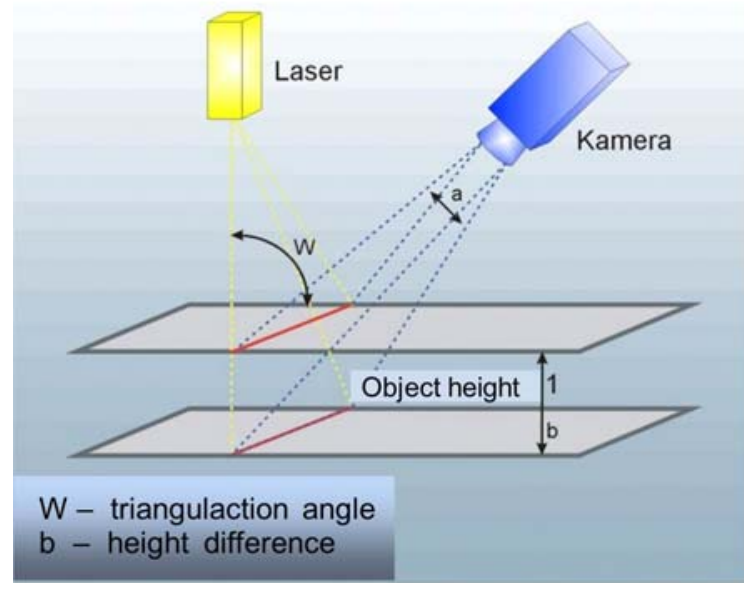

Fig. 1. Optical triangulation principle.

The Vitus Smart 3D body scanner allows to scan an object in the area of 1,200 x $800 \mathrm{~mm}$ and 2100 $\mathrm{mm}$ in height. Scanning is performed by the system of 8 cameras and lasts 10 seconds, whereby 500,000 to 600,000 spatial coordinates of the scanned body are extracted. Data processing takes about 40 seconds, and then using the software package ScanWorx V 2.7.2. human body measurements necessary for the implementation in the computer program for the alteration of the garment pattern according to the determined measurements are extracted. The ScanWorx software package offers the possibility of correcting the obtained body measurements, as well as adding new ones. Using video cameras, laser beam position on the object is detected outside the fixed angle. Considering the triangulation angle and the formation of a stationary optical form, the position of an object in the direction of the $x$ and $y$ coordinates can be calculated. To achieve the third dimension of the object, the triangulation sensor is moved in precisely defined steps in the direction of the $z$ axis. Using information on the distance between steps, the shape of an object or body part is scanned piece by piece in layers. The VITUS Smart 3D scanner uses triangulation sensors targeted toward the body from different directions (total $360^{\circ}$ ) for scanning the human body in one pass. 
To ensure the precise determination of body measurements, it is necessary to periodically check and if necessary carry out calibration of the scanner. Additionally, the person to be scanned should stand in a proper upright posture during scanning, dressed in underwear of bright color tight to the body. The hair should be tied behind the back of the head, and for the most accurate measurement results, it is desirable to wear a swim cap. Also, it is necessary to remove the jewelry.

The automatic computer procedure to determine body measurements for a 3D group of points is used to determine the values for a total of 85 body measurements, and the automatic computer-based measurements are used to determine the distance between the computer-defined positions of measuring points and by measuring the girths of individual body parts. The results of the automatic computer-based determination of body measurements are generated in a table after the measurements where a graphic representation of the position of body measurements is given for each determined value. Out of the total number of determined measurements, some measurements have been determined separately for the left and right side of the body, as it is for example the length of the arms and shoulders, then the length of the left and right shoulder, the values of the angles that determine the slope of the shoulder, crotch length. Separately were also measured the values of the individual body parts, such as the circumference of the left and right upper arm, wrist, then the circumference of the left and right leg at the height of the thigh, knee, lower leg and ankle. In addition to the measurements determined by the automatic measurement, all possible additional measurements, if necessary, can be determined by the interactive measurement [8].

\subsection{Interactive adjustment of the parametric body model according to individual measurements}

Interactive adjustment of parametric model implies entering the values of individual measurements previously determined by use of the 3D body scanner. When adjusting avatars in a CAD system to the measurements determined with $3 \mathrm{D}$ body scanning special attention should be paid to the position of the anthropometric measuring landmarks that should be in harmony with the ISO and EN standards that prescribe a way of determining body measurements for the needs of garment construction [9]. If the adjustment position of an individual body measurement on the avatar, which uses 3D CAD system, is different from the measurement position and body measurements on a scanned body model, it is necessary to perform an interactive measurement of the selected measurement on the scanned body model, namely at the position defined by avatar adjustment, fig. 2 .
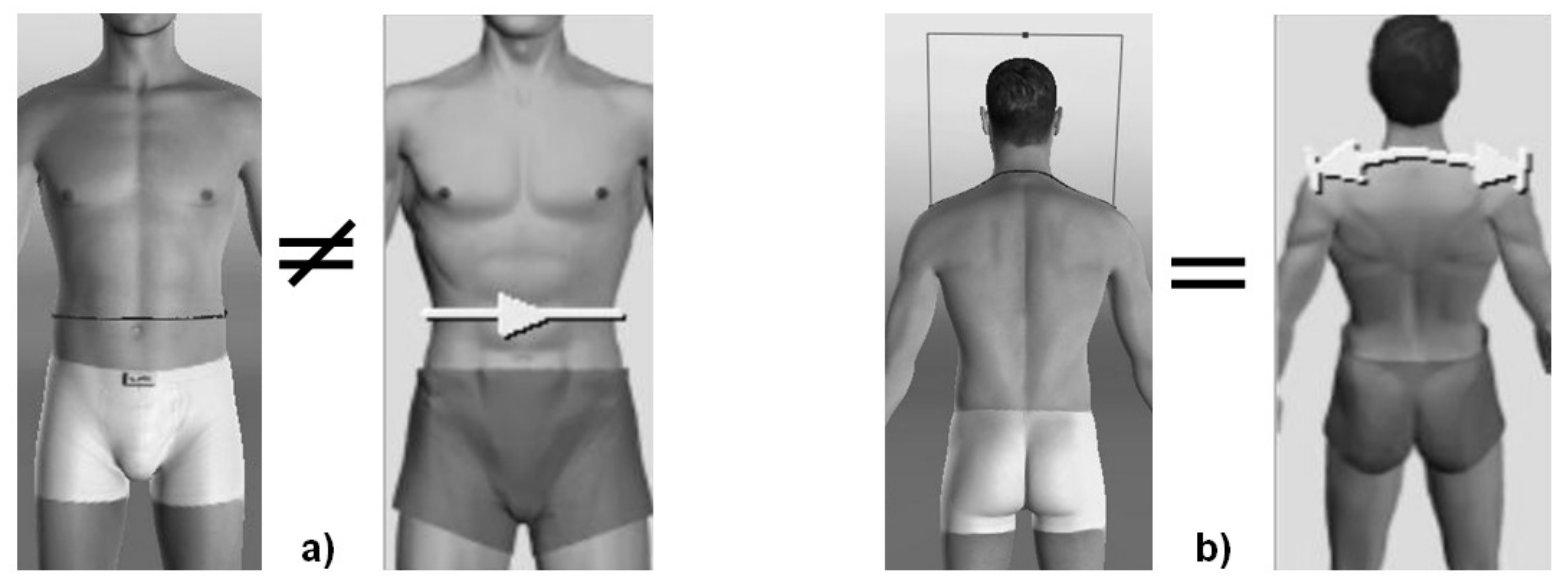

Fig. 2. Comparison of selected measurements adjustment position on computer parametric 3D model with the measurement position of the same body measurement on a model obtained by $3 D$ body scanning: a) incompatibile positions of waist circumference, b) compatible positions of shoulder width.

Table 1 lists the measurements necessary to adjust the avatar used by the Optitex CAD system and the corresponding measurements obtained by automatic measurement of 3D scanned body model. Apart from a smaller number of measurements whose adjustment position of the parametric model differs from the position of the corresponding measurement on the scanned body model, some of the measurements cannot be determined with automatic measurement, and it is necessary to perform interactive measurements of the scanned model. Alternatively, some measurements can be determined from the difference of the values of two relevant determined measurements, fig. 3. 
Table 1. Compatibility of avatar and scanned body anthropometric data.

\begin{tabular}{|c|c|c|c|}
\hline & $\begin{array}{c}\text { Avatar } \\
\text { anthropometric } \\
\text { characteristics }\end{array}$ & $\begin{array}{l}\text { Automatic measures of } \\
\text { scanned model }\end{array}$ & comment \\
\hline \multirow{21}{*}{$\frac{\mathscr{U}}{\infty}$} & Size (Underchest) & Midriff girth & \multirow{3}{*}{$\begin{array}{l}\text { Suitable measuring } \\
\text { position }\end{array}$} \\
\hline & Height & Body height & \\
\hline & Cervical height & Neck height & \\
\hline & Posture & - & \multirow{3}{*}{ Interactive adjustment } \\
\hline & Seat Prominence & - & \\
\hline & $\begin{array}{l}\text { Upper Body } \\
\text { Prominence }\end{array}$ & - & \\
\hline & Body Width & Breast width & $\begin{array}{c}\text { Suitable measuring } \\
\text { position }\end{array}$ \\
\hline & Body Depth & $\begin{array}{l}\text { Distance front breast to vertical - } \\
\text { distance back breast to vertical }\end{array}$ & \multirow{2}{*}{$\begin{array}{l}\text { Difference between two } \\
\text { measurements }\end{array}$} \\
\hline & Belly (Depth) & $\begin{array}{l}\text { Distance front belly to vertical - } \\
\text { distance back belly to vertical }\end{array}$ & \\
\hline & Weight Balance & - & \multirow{12}{*}{ Interactive adjustment } \\
\hline & Muscles & - & \\
\hline & Arms Mass & - & \\
\hline & Trapezius & - & \\
\hline & Buttocks Bump & - & \\
\hline & Buttocks Type & - & \\
\hline & Crotch Width & - & \\
\hline & Crotch Size & - & \\
\hline & Front Thigh Curve & - & \\
\hline & Back Thigh Curve & - & \\
\hline & Back Waist Length & - & \\
\hline & Front Waist Length & - & \\
\hline \multirow{11}{*}{ 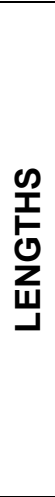 } & Cross Shoulders & Cross shoulders over neck & \multirow{6}{*}{$\begin{array}{l}\text { Suitable measuring } \\
\text { position }\end{array}$} \\
\hline & Outseam & Outseam & \\
\hline & Inseam & Inseam & \\
\hline & Hip Height & Hip height & \\
\hline & Waist to Hip & Waist to hip & \\
\hline & Arms Length & Arm length & \\
\hline & Knee Height & Waist height - waist to knee & $\begin{array}{c}\text { Difference between two } \\
\text { measurements }\end{array}$ \\
\hline & Waist to Hip & Waist to hip - unsuitable position & \multirow{4}{*}{$\begin{array}{c}\text { MEASURING ON } \\
\text { SCANNED BODY MODEL }\end{array}$} \\
\hline & UnderChest Height & - & \\
\hline & High Hip Height & - & \\
\hline & Armscye Depth & - & \\
\hline \multirow{17}{*}{ 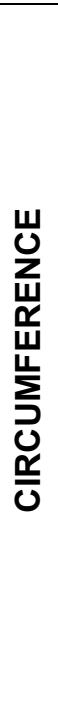 } & UnderChest & Midriff girth & \multirow{12}{*}{$\begin{array}{l}\text { Suitable measuring } \\
\text { position }\end{array}$} \\
\hline & Waist & Waist girth & \\
\hline & Hip & Hip girth & \\
\hline & Chest & Breast girth & \\
\hline & High Hip & High hip girth & \\
\hline & Thigh & Thigh girth & \\
\hline & Knee & Knee girth & \\
\hline & Ankle & Ankle girth & \\
\hline & Upper Biceps & Upper arm girth & \\
\hline & Wrist & Wrist girth & \\
\hline & Neck & Mid neck girth & \\
\hline & Base Neck & Neck girth & \\
\hline & Waist & Waist girth - unsuitable position & \multirow{5}{*}{$\begin{array}{c}\text { INTERACTIVE } \\
\text { MEASURING ON } \\
\text { SCANNED BODY MODEL }\end{array}$} \\
\hline & Low Thigh & - & \\
\hline & Calf & - & \\
\hline & Biceps & - & \\
\hline & Elbow & - & \\
\hline
\end{tabular}




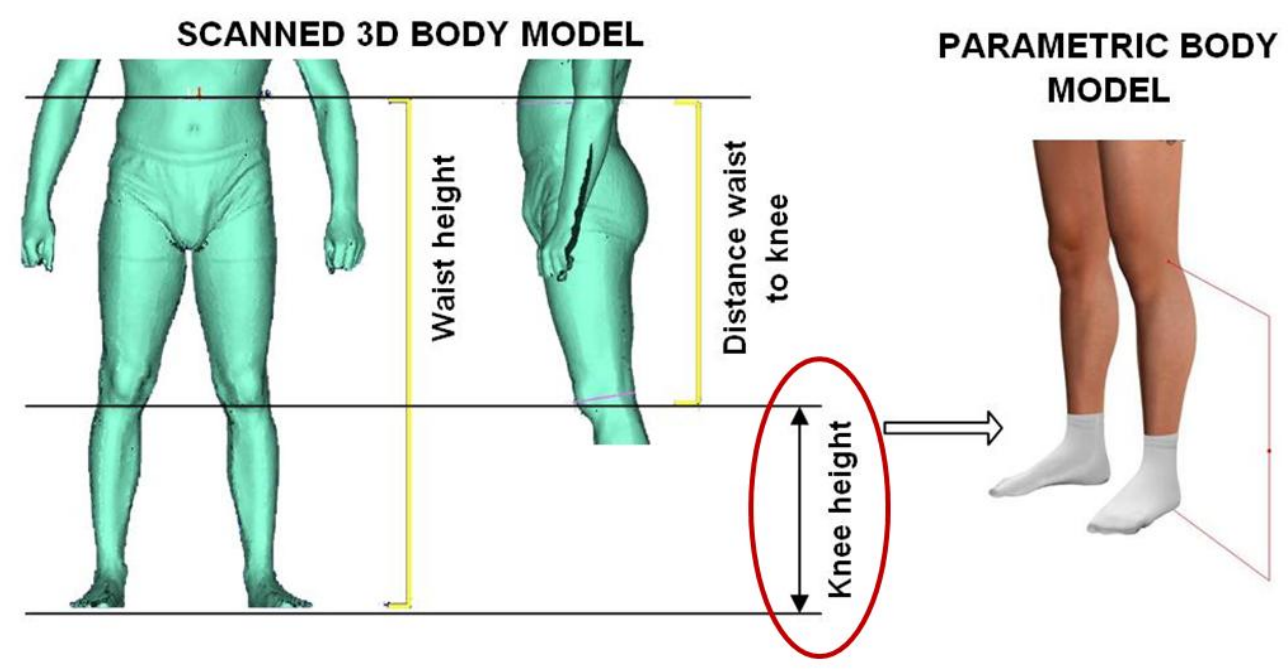

Fig. 3. Definition of the values of the knee height on the parametric body model by means of the difference between two body heights found by $3 D$ scanning.

The adjustment of the measurements related to the posture and shape of the parametric model is performed based on the analysis of the posture and shape of the scanned body model, whereby spine curvature, shoulder position, development of muscle mass in muscular areas, shape and position of the buttocks, etc. are defined. These measurements cannot be accurately quantified, but are adjusted using a slider within the boundaries of significance of certain body characteristics that system defines, fig. 4 .

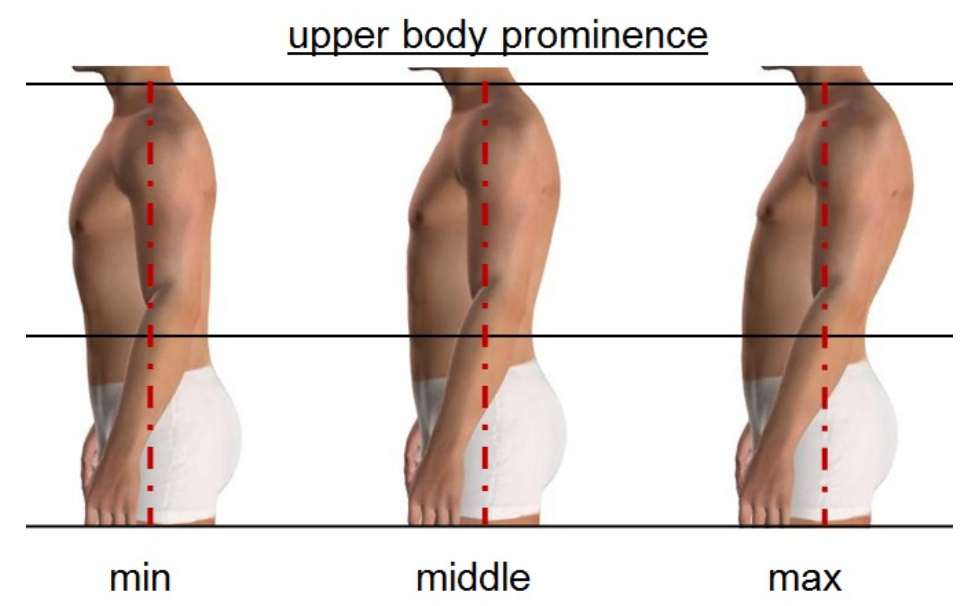

Fig. 4. Interactive adjustment of avatar torso inclination.

\subsection{Automatic adjustment of the parametric body model creating a file of measurements obtained by 3D scanning}

The adjustment of the parametric body model can also be performed automatically, by implementing the measurement file, whereby only partial adjustment is performed with respect to a large number of measurements on parametric models that are not precisely quantified. In this context, according to table 1 , it is initially necessary to interactively adjust anthropometric characteristics of the body on the avatar, in line with the analysis of posture, since in case of the same body measurement the value of each body girth can be different, depending on the shape of each body segment. Then the program downloads the file of body measurements determined using 3D body scanner according to which automatic avatar adjustment is performed. When creating a file of measurements, the sequence of all necessary measurements must be correlated with the sequence of the measurements defined in the program for interactive adjustment. The program sets certain limits according to the value of the main body measurements (underchest girth) with the possibility of modifying the width of other body parts [10]. 


\subsection{Import of the computer body model obtained by 3D scanning into the CAD system}

Besides the use of avatars, the CAD system enables the implementation of $3 D$ body models created in one of commercial systems for 3D modeling and obtained by 3D body scanning. When implementing the body model obtained by 3D scanning, it is necessary to convert the model into a format of the record that supports the CAD system, and be careful about compatibility of the local coordinate system within the 3D object and the measuring units used by the system. The figure shows the positions of the body for 3D scanning prescribed by the standard ISO 20 685, fig. 5a; however, for later simulation and fit testing of clothing in the CAD system on the 3D body model, the body posture in 3D scanning should be as shown in fig. $5 \mathrm{~b}$. Additionally, scanning can be performed with a body position as shown in fig. 6 ., or similar, for the purposes of presentation of garment collections. It is necessary that the upper and lower body extremities are not held together or along the trunk in order to perform simulations of each article of clothing.

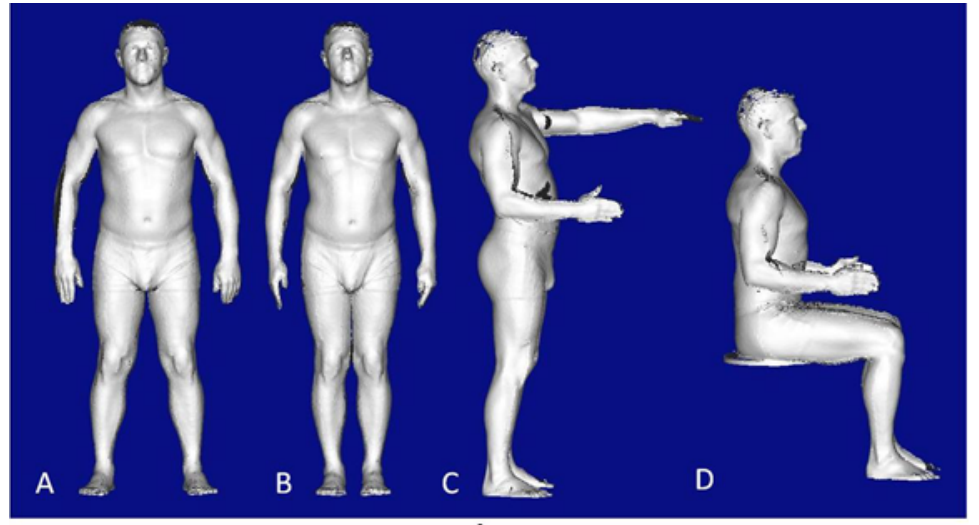

a)

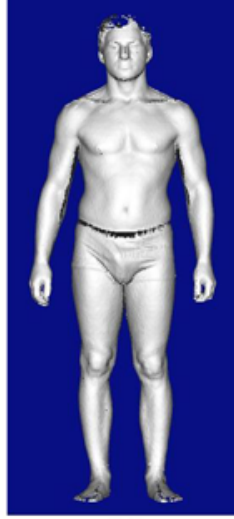

b)

Fig. 5. Body postures for computer 3D scanning.
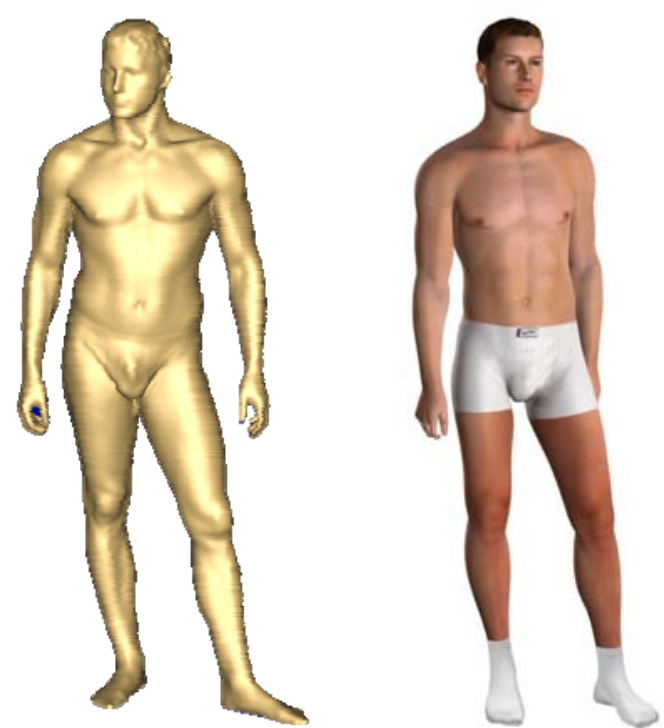

Fig. 6. Body postures for fashion visualizations of clothing.

\section{Experimental part}

Using 3D laser scanner body Vitus Smart and accompanying computer program ScanWorx V 2.7.2. body scan, computer analysis and anthropometric measurements of the body of male test subjects on a sample of 20 individuals of normal stature, but different body posture and shapes of individual body parts, was performed. Such a pattern is defined for the purpose of investigation of the influence of characteristic physical scale in the form of individual body parts, as defined by the same physical volume, in different subjects, shape or cross section of the relevant parts of the body can be very different. Such a pattern is defined for the purpose of investigation of the influence of characteristic body circumferences on the shapes of individual body parts, as for the same defined body 
circumference in different subjects, the shape or cross section of the relevant body part can be very different. Based on the identified data the possibilities of avatar adjustment in a CAD system using three previously described methods were investigated, and the following paragraphs of the paper present the avatar adjustment using all three methods using the example of one of the subjects, with the aim of verifying the mentioned methods. Finally, verification of the applicability of adjusted avatars was made by computer simulation of the same garment, of the appropriate clothing size, on all three computer body models.

\subsection{Interactive avatar adjustment in 3D CAD system}

Based on the previously determined measurements obtained by automatic measurement of the scanned body models, and interactive measuring of unadjusted measurements and missing measurements (table 1), interactive avatar adjustment was performed. With respect to the impact of measurement adjustments that relate to body shape and the measurements of characteristic circumferences on each other, first the adjustment of body posture and shape was performed, posture and body shape after which the amounts of individual characteristic body measurements were defined in a specified order.

\subsubsection{Adjustment of body posture and shape}

The posture of the parametric body model was determined with three measurements by means of which, according to the performed analysis of the body posture of the scanned model, spine curvature, position of hips and torso inclination in relation to the central body axis, fig. 7., is defined.

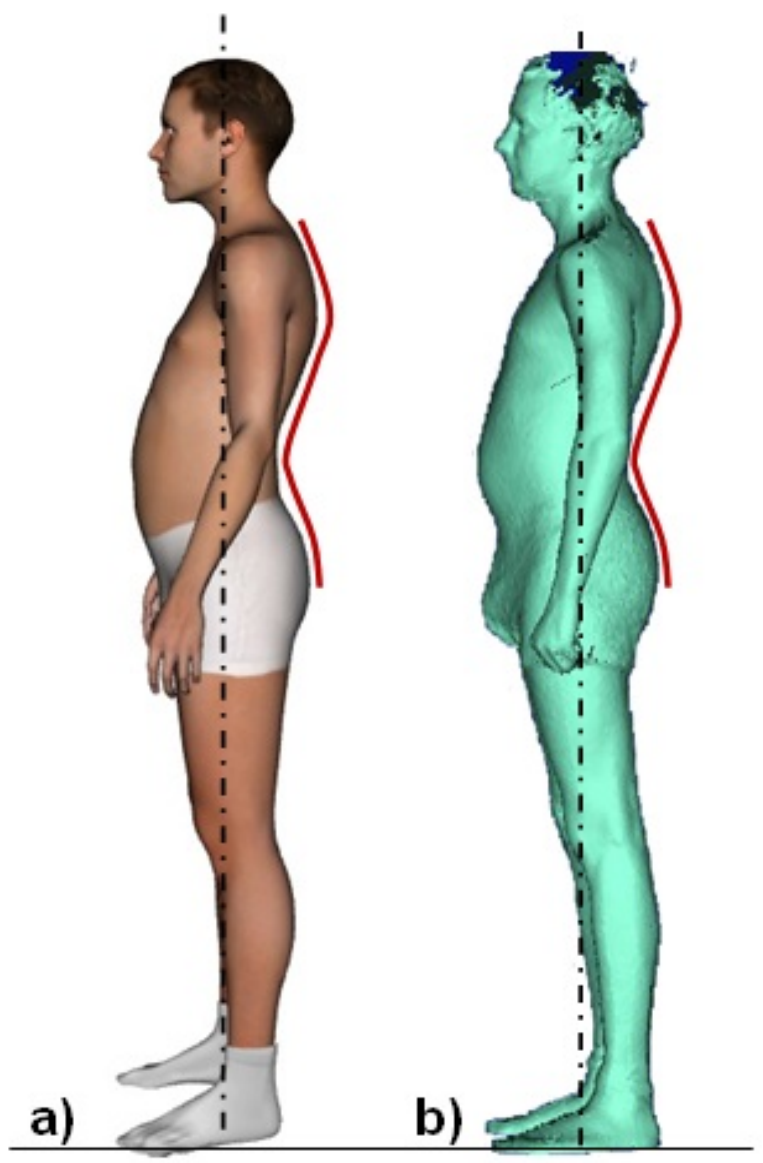

Fig. 7. Adjustment of body posture: a) avatar, b) scanned body model.

Measurements such as the depth of the body of the characteristic circumferences can be calculated from the difference of the distance between corresponding points of the given circumference from the given vertical axis set in the computer program for 3D body scanning and measurements, fig. 8 . 


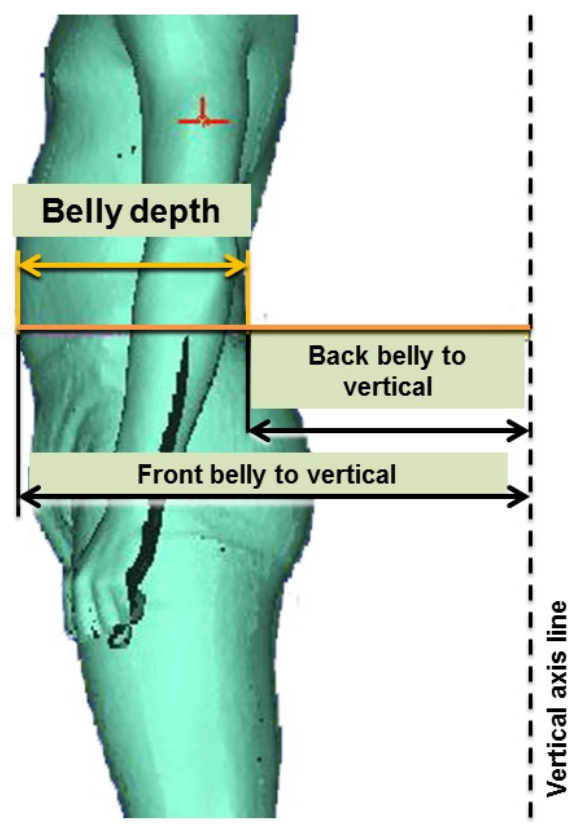

Fig. 8. Calculation of body depth.

3.1.2. Harmonization of measurement positions and interactive measurement of the scanned model The interactive measurement of the scanned body model was performed using measuring tools of the software package ScanWorks V 3.0.1 made by Human Solutions. The values of eight measurements were determined, whereby two measurements have an unadjusted position on the body in relation to the avatar, while the other six measurements were not determined with automatic measuring, fig. 9.

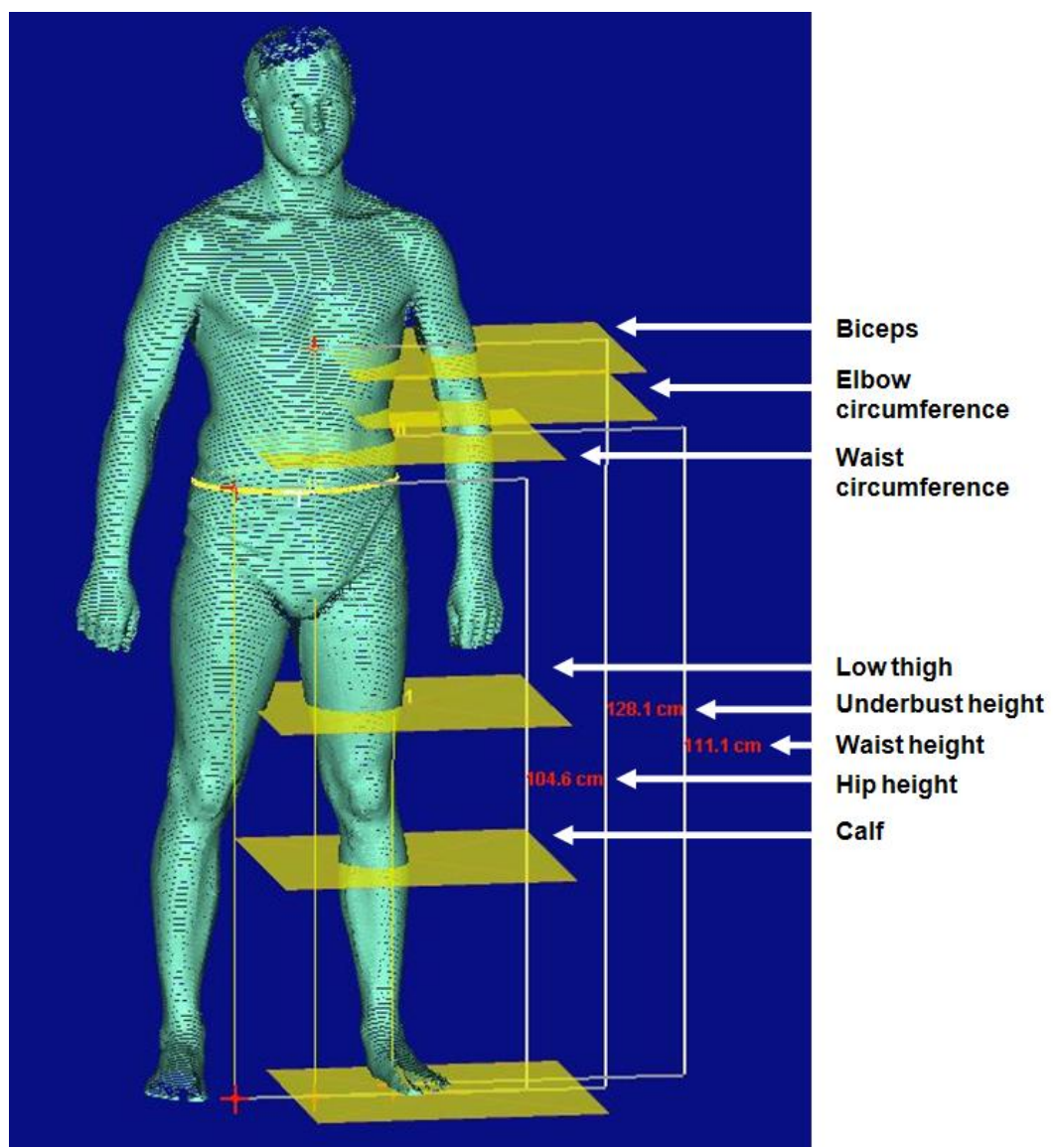

Fig. 9. Interactive computer measurement of the scanned 3D body model according to the positions of the same measurements on the avatar. 
Interactive measurement of the required body heights was performed by measuring the vertical distance of a point at a given height in relation to the lower transverse plane, while the girths were obtained by cutting the model with the transverse planes at the given positions and by determining the circumference of the cross-sections, fig. 10.

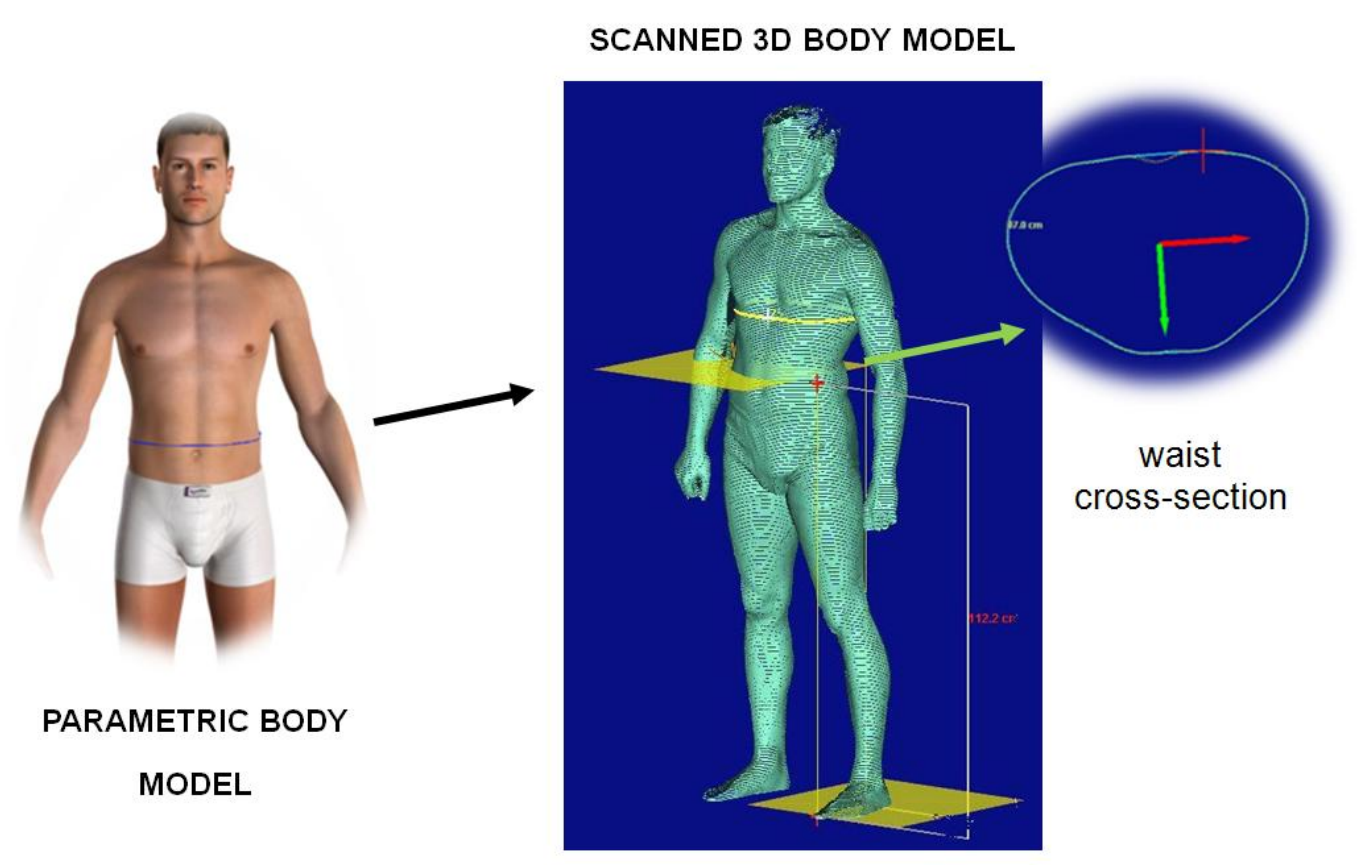

Fig. 10. Harmonization of the position of the body measurement of waist girth and determination of the circumference of the cross-section and the waist height at the given position.

\subsection{Creating data files of measurements for automatic avatar adjustment}

A data file of measurements for automatic avatar adjustment was created on the basis of the obtained measurement results, whereby a set of 22 body measurements was selected for the adjustment, while the other body measurements will be adjusted in correlation with the defined alterations. The adjustment of the posture of the parametric body model was initially performed in the program according to the performed analysis of body posture of the model determined by scanning. Then, by downloading the data file of measurements the automatic adjustment of the measurements of the parametric body model was performed. In this way, the adjustment of the parametric body model according to the body model obtained by body scanning was performed much faster than the interactive adjustment of the model by gradual alterations and measurement adjustments. The parametric model of the body was adjusted in the following measurements: body height, height of the $7^{\text {th }}$ cervical vertebra, height of the underchest girth, side seam to the waist, crotch length, knee height, shoulder width, lower neck girth, mid neck girth, chest girth, underchest girth, waist girth, hip girth, high hip girth, thigh girth, knee girth, calf girth, ankle girth, lower biceps girth, upper biceps girth, and wrist girth.

\subsection{Implementation of the computer body model obtained by 3D scanning}

The computer-based body model obtained using 3D body scanner was converted into a format of the record suitable for the implementation of the computer model into 3D CAD system. The lack of scanned computer models results from the non-closeness of the point cloud describing the body. The cause of this is the lack of data on the coordinates of the points in the shaded areas on the body such as armpits, inseam, etc. In this sense, using the tools of the software package ScanWorks V 3.0.1. subsequent processing of the scanned model was performed in order to close the area of the computer body model, fig. 11. After the implementation of such a body model in the CAD system it is no longer necessary to perform any adjustments, but as such it is used for garment simulation. 


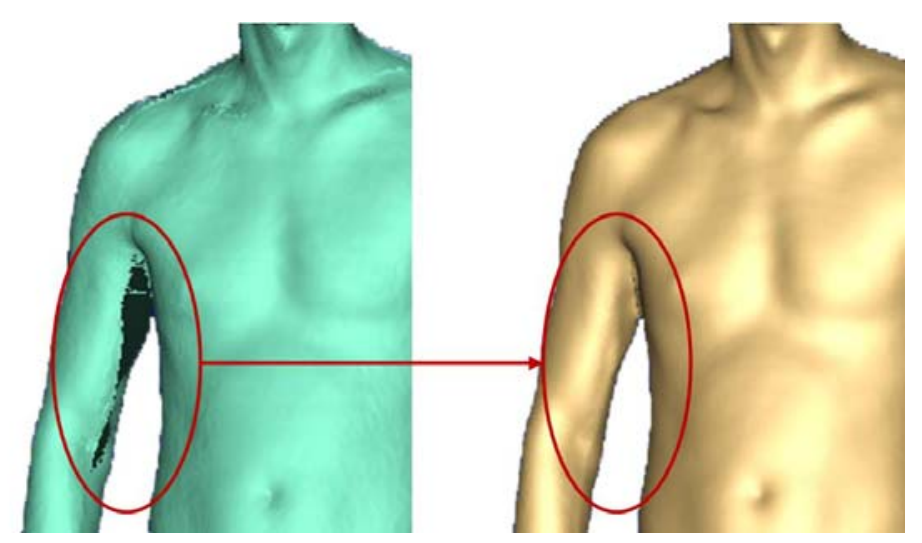

Fig. 11. Computer-based closing the area of the scanned body model.

\subsection{Simulation of garment model}

A simulation was performed on each of the 3D models for the purpose of verification of computer body models and the assessment of the fit of the selected garment. For this purpose the classic pattern of a men's jacket in size 100 was selected, corresponding to the measurements of the scanned test subject, and was also designed using the CAD system made by OptiTex, which defines all the parameters needed to run the simulation [11].

\section{Results}

Below are the results of the computer avatar adjustment for the first two methods, and the processed computer model of the body imported into the CAD system using the third method, fig. 12. and 13. The figure shows minor differences, which were determined by measuring models (approx. $1 \mathrm{~cm}$ in the value of a measurement) between the adjusted avatars using the first and second method, and the processed computer body models. Also on the computer body model the asymmetry of the body in the shoulder slope is visible, while in case of the avatar the adjustment was performed symmetrically on both sides of the body. As such, all body models are suitable for the simulation of the garment in the selected size with the purpose of the verification of pattern fit, fig. 14 .

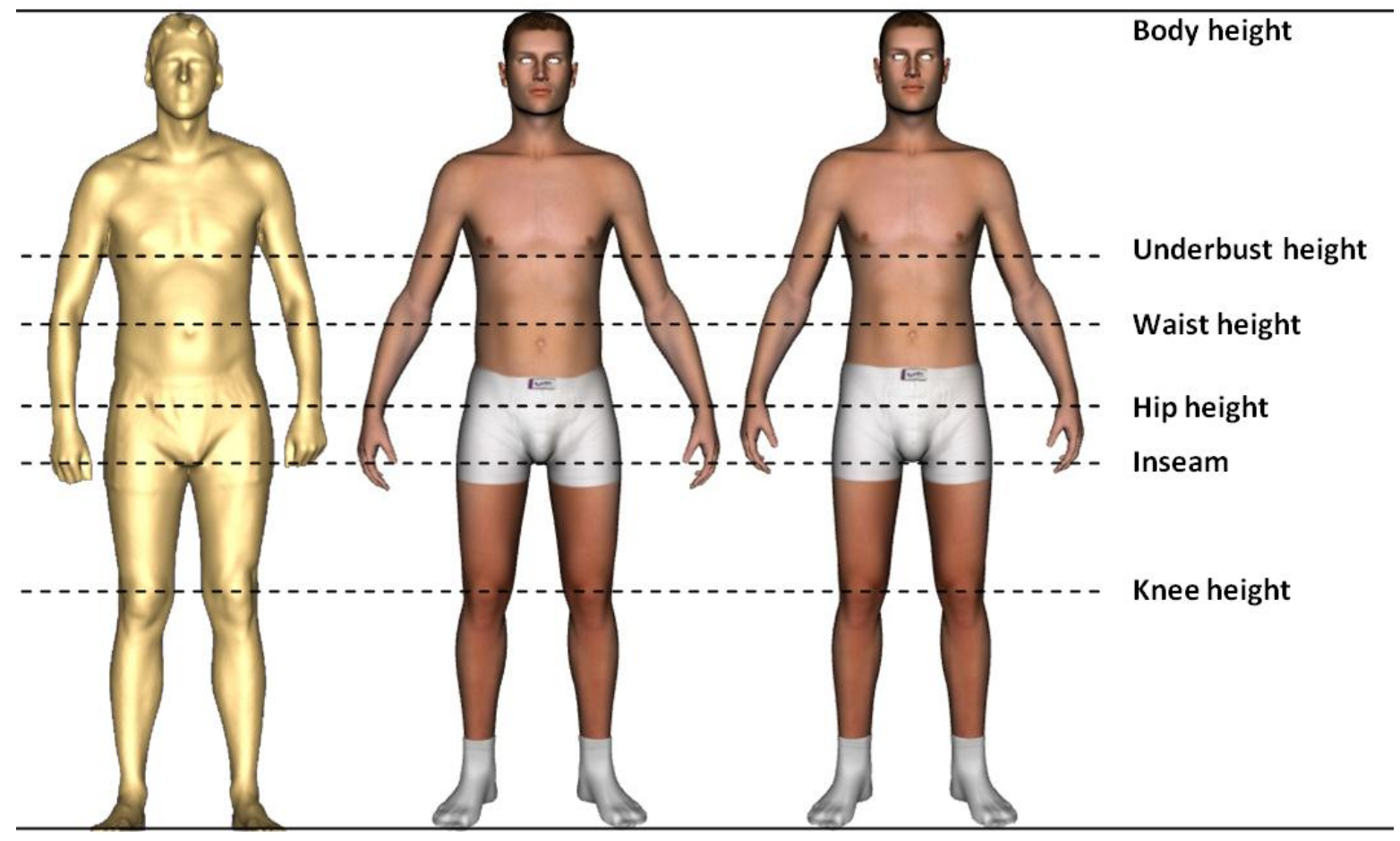

a)

b)

c)

Fig. 12. Computer-based body models: a) scanned computer-based body model, b) avatar adjusted using the interactive method, c) avatar adjusted using the automatic method. 


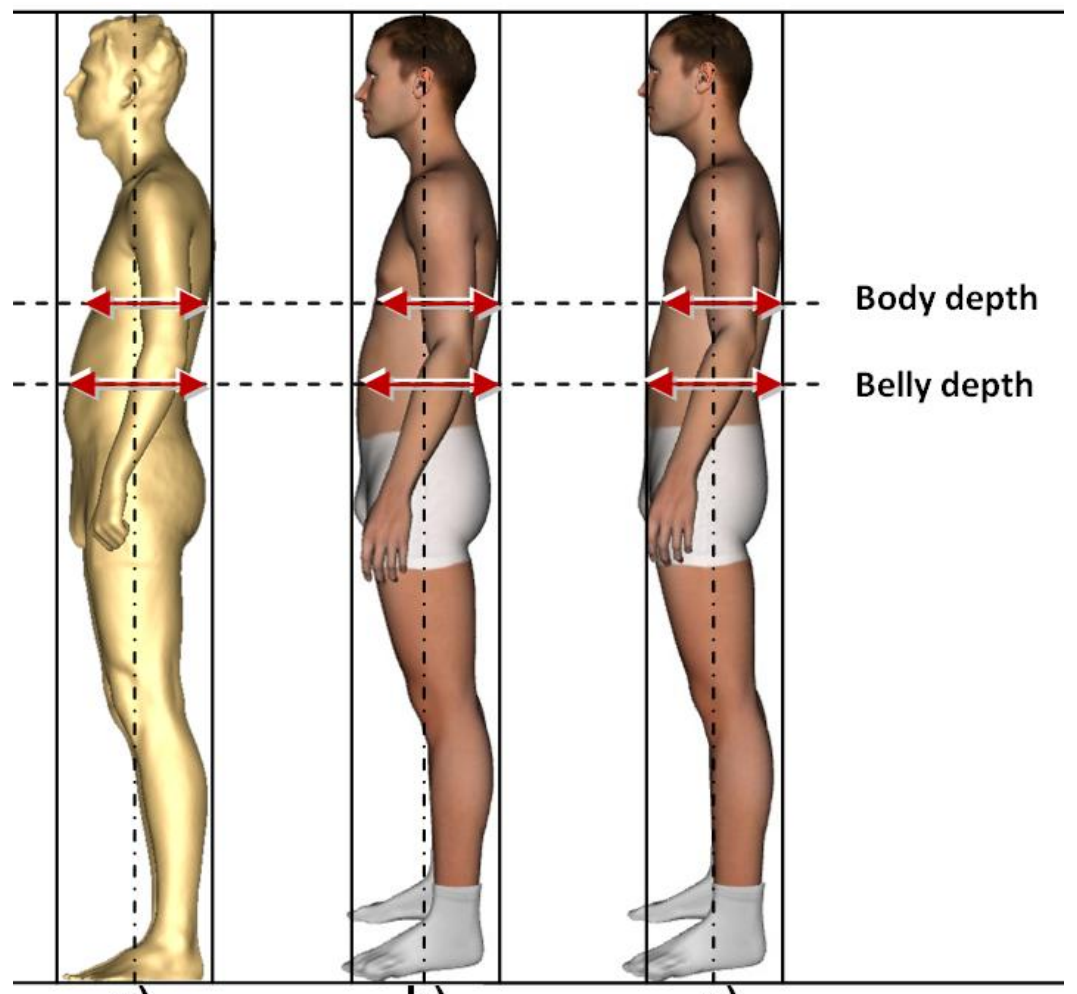

a)

b)

c)

Fig. 13. Comparison of body posture and depth: a) scanned computer-based body model, b) avatar adjusted using the interactive method, c) avatar adjusted using the automatic method.

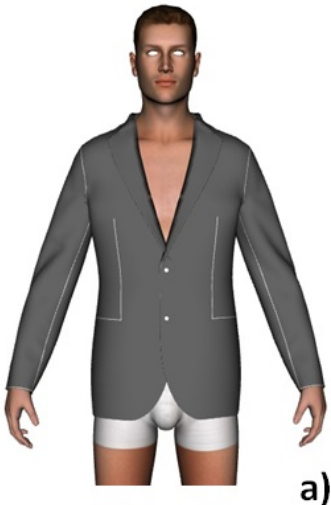

a)
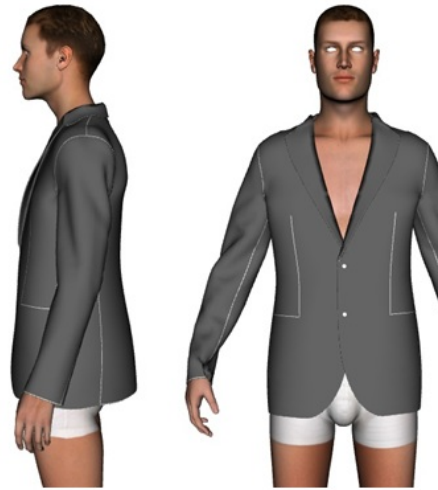

b)

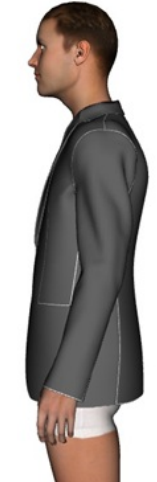

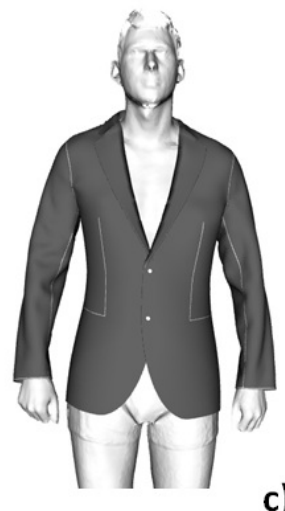

c)

Fig. 14. Simulation results of a men's jacket in appropriate garment size on computer-based body models obtained using different methods: a) avatar adjusted with the interactive method, b) avatar adjusted with the automatic method, c) scanned computer-based body model.

\section{Conclusions}

The research described in this paper was conducted with the aim of finding the possibility to implement data on anthropometric body characteristics identified using the 3D body scanner in the CAD system for design and 3D garment simulation. Data implementation was performed using three methods. Using the first method allowing interactive adjustment of anthropometric avatar properties in the CAD system the gradual adjustment of body posture, development of individual body parts and avatar measurements was performed so that ultimately the avatar should be adjusted as good as possible to anthropometric body properties of the scanned person. The advantage of interactive avatar adjustments lies in a relatively simple adjustment of the characteristic lengths on the body, while achieving the harmonization of body shape and the measurements of characteristic girths requires multiple adjustments of measurements which are correlated with each other. Thus, the complete avatar adjustment is longer and requires a higher level of knowledge and experience of the user, but 
ultimately the avatar can be adjusted highly realistic and accurate enough for the simulation of the scanned person's body in the CAD system. The application of the second method that involves the systematic organization of body measurements determined using the 3D body scanner in the file appropriate for the implementation in a CAD system and partially automated avatar adjustment also has its advantages and disadvantages. As an advantage of this method shorter necessary time can be emphasized compared to the first method to adjust the avatar. However, this method also requires the partial interactive avatar adjustment since it is first necessary to interactively adjust the posture and shape of certain body parts, and only after this action avatar measurements can be adjusted automatically by downloading the measurement file.

Both methods assume the complete symmetry of the body since the adjustment is performed identically on the left and right side of the body. The third method of direct downloading the computer processed and scanned body model is the fastest, whereby there is no need to analyze the body by measuring the body and processing the measurement data, but the model as such represents a very accurate replica of the scanned test subject's body and can be used for garment simulation.

The verification of all three methods was performed with the 3D simulation model of a garment where it was found that all three methods are acceptable for testing the prototype model of a garment with the aim of evaluating its fit. Because of its complexity, the first method would be still more appropriate for avatar adjustment according to the garment size system, whereby the body measurements on the avatar would be adjusted to mean measurement values, and such avatars could be used to test the collection of clothes in terms of industrial mass production. The second method would be suitable for avatar adjustment in terms of industrial production of clothes made to measure, but for people of normal body posture and with no deviation from the normal proportions. However, only in case of the third method it is to possible to evaluate the need for additional adjustment of the garment pattern if there is a greater asymmetry of the body and deviations from normal body proportions and posture. In this sense, this method is most suitable for the industrial production of clothes made to measure.

\section{References}

1. Fan J., Yu W., Hunter L. (2004): "Clothing appearance and fit: Science and Technology", Woodhead Publishing Limited and The Textile Institute, ISBN 185573745 0, Cambridge England

2. D'Apuzzo, N., (2009): "Recent advances in 3D full body scanning with applications to fashion and apparel", Proceedings of $9^{\text {th }}$ Conference on Optical 3D Measurement Techniques 2009, Vienna, Austria

3. Chun, J., Oh, S., (2004): "3D Body Scanning Posture to Collect Anthropometric Data for Garment Making", Journal of Asian Regional, Association for Home Economics, Vol.11, No.4, pp.301-307.

4. Simmons, K. and Istook, C., (2003): "Body measurement techniques: Comparing 3D body-scanning and anthropometric methods for apparel applications" Journal of Fashion Marketing and Management, Vol.7, No.3, pp.306-332.

5. Ujevic, D., Szirovicza, L. and et.al., (2010): "Comparison of Conventional and Computerised Human Body Measurement Methods", Proceedings of $5^{\text {th }}$ ITC\&DC - Magic World of Textiles, Dubrovnik, Croatia, p.p.523-528.

6. Zhang, X., (2008): "Anthropometry and Clothing Engineering", Proceedings of TBIS, Hong Kong, p.p.828-832 doi:10.3993/tbis2008135, www.tbisociety.org [pristupljeno: 20.5.2012.]

7. Ujević, D., Petrak S., Hrastinski M. and Mahnic M., (2012): "Development of the garment size system and computer-based body models", Journal of Textiles and Engineer, Vol. 19, No.85, pp. $35-40$.

8. Ujevic, D., Petrak, S. and et.al., (2009): "Conventional and Computer-controlled Methods In Anthropometry under Consideration", Proceedings of $7^{\text {th }}$ International Scientific Conference on Production Engineering, Rim, p.p.152-157.

9. 3D Scanning methodologies for internationally compatible anthropometric databases, international standard ISO/FDIS 20685:2010.

10. Petrak, S. and Mahnic, M., (2012): "Implementation of modern computer systems in the process of industrial design and garment construction", Proceedings of $5^{\text {th }}$ Scientific-professional Symposium Textile Science and Economy, TZG, Zagreb, Croatia, pp. 25-36.

11. Mahnic, M. and Petrak, S., (2012): "Investigation of the fit of computer-based parametric garment prototypes", Proceedings of $12^{\text {th }}$ World textile conference AUTEX 2012, Zadar, Croatia, pp.961-966. 